\title{
Análisis de fragancias florales por GC-MS
}

\author{
Elena E. Stashenko*, Jairo René Martínez \\ Centro de Cromatografía y Espectrometría de Masas - CROM-MASS, Centro de Investigación de Excelencia - CENIVAM, \\ Escuela de Química, Universidad Industrial de Santander - UIS, Bucaramanga, Santander, Colombia \\ e-mail:elena@tucan.uis.edu.co
}

\section{Resumen}

Se describen diversas características químicas de las fragancias florales y se presentan ejemplos de aplicación de los métodos de espacio de cabeza, destilativos y extractivos más usados comúnmente para el aislamiento de estas mezclas de compuestos orgánicos volátiles cuya concentración varía entre picogramos y microgramos por litro. Para el análisis GC-MS de las fragancias florales se presentan recomendaciones sobre su separación, detección e identificación, acompañadas de ejemplos.

\section{Palabras clave}

Fragancia floral; microextracción en fase sólida; cromatografía de gases acoplada a espectrometría de masas; compuestos orgánicos volátiles.

\section{GC-MS analysis of floral fragrance}

\section{Abstract}

The diverse chemical features of floral fragrances are described and examples of the application of headspace, distillative and extraction methods most commonly used to isolate these mixtures of volatile organic compounds whose concentration varies between picograms and micrograms per liter are presented. Regarding the GC-MS analysis of floral fragrances, recommendations about their separation, detection and identification are presented together with examples.

Keywords

Floral scent; solid-phase microextraction; gas chromatography coupled to mass spectrometry; volatile organic compounds. 


\section{Introducción}

Uno de los campos de estudio de productos naturales comprende un área muy interesante: la obtención y el análisis de las fragancias florales, que pueden ser monitoreadas tanto in vivo, como ex vivo ${ }^{[1]}$. El estudio de metabolitos secundarios volátiles, que emanan las flores, es importante en muchas áreas de las ciencias biológicas y químicas, en agricultura, para el control de plagas, en el estudio de interacciones planta-insecto, alelopatía, en ciencias analíticas (preparación de muestras y cromatografía), en industrias farmacéutica, de perfumes y cosmética, sabores y fragancias, entre otras. El interés creciente en este campo de investigación se refleja en que en los últimos 20 años, el número de artículos científicos publicados por año sobre el tema, creció de 7 (1993) a $158(2012)^{[2]}$.

Las fragancias florales son mezclas complejas, producto del metabolismo de una planta en florecimiento; están compuestas por centenares de moléculas de diferente origen bioquímico, con distintas características físico-químicas (polaridad, volatilidad, solubilidad); contienen diversos grupos funcionales (hidrocarburos, alcoholes, aldehídos, cetonas, ácidos, ésteres, etc.) y pueden encontrarse en concentraciones muy disímiles (desde partes por trillón, ppt, hasta partes por millón, $p p m)$. Son sustancias predominantemente lipofílicas, con peso molecular menor de $300 \mathrm{Da}$, apolares o medianamente polares, con presión de vapor alta. La nariz humana puede ser más sensible -que un sistema de detección cromatográfico-, a algunas sustancias de fragancia floral, presentes a nivel de trazas; por ello, es necesario llevar a cabo el proceso de extracción y concentración de la fragancia floral de tal manera, que sus componentes sean detectables y puedan ser identificados. Esto constituye un reto analítico muy grande. Hoy en día, este reto se resuelve aplicando diferentes estrategias: técnicas de extracción en espacio de cabeza (headspace), métodos destilativos, extractivos con solventes, y en la superficie activa, i.e., procesos de adsorción/desorción térmica usando adsorbentes con diferentes características físico-químicas ${ }^{[3,4]}$. Para el análisis instrumental de las fracciones volátiles y extractos se usa la cromatografía de gases (GC) en versiones monodimensional o bidimensional, GCxGC, en columnas capilares de diferentes polaridades y sistemas de detección tanto universales (detector de ionización en llama, FID; detector selectivo de masas, MSD, por sus siglas en inglés), como selectivos (detector selectivo de nitrógeno y fósforo, NPD; detector fotométrico de llama, FPD, para registrar compuestos nitrogenados o azufrados, respectivamente) y muy específicos (electroantenografía, nariz electrónica, etc. $)^{[5]}$.

\section{Composición química de las fragancias florales}

Muchos y muy diversos compuestos han sido detectados e identificados en las fragancias florales. Más de 1700 han sido registrados en una multitud diversa de flores estudiadas ${ }^{[6,7]}$. Los grupos principales de compuestos químicos encontrados en emisiones florales comprenden hidrocarburos (saturados, cíclicos y olefínicos); terpenos, básicamente, monoterpenoides; y bencenoides y fenilpropanoides, entre otros compuestos oxigenados de naturaleza mixta, alcoholes, aldehídos, éteres, ésteres y sustancias que incorporan heteroátomos, azufre o nitrógeno.

La composición de la fragancia floral es uno de los aspectos más relevantes y variados del fenotipo de las plantas. La función básica de la fragancia floral es promover la polinización cruzada, que es un proceso vital en el ciclo de vida de la mayoría de las plantas ${ }^{[1]}$. El conocimiento de la composición de la fragancia floral es impor- 
tante para entender la interacción planta-insecto, las estrategias químicas para atraer los polinizadores, pero también para disuadir a los herbívoros y enfrentar a los patógenos, para adaptarse a diferentes estreses abióticos; para estudiar las rutas bioquímicas de formación de metabolitos secundarios en una planta, su capacidad de adaptación y la evolución biológica. También, es de interés práctico conocer la composición floral como fuente de inspiración para crear nuevas fragancias y mezclas odoríferas, que se usan en las industrias de cosméticos, perfumes, productos de higiene personal o en la aromaterapia.

Las fragancias florales de diferentes plantas, a pesar de tener un olor diferente y a menudo, distintivo y diferenciador entre especies y variedades vegetales, poseen muchos compuestos comunes. Entre estos, conforman un grupo grande los terpenoides: monoterpenos, sesquiterpenos, diterpenos y terpenos irregulares y sus análogos oxigenados. Entre los más frecuentes, figuran monoterpenos oxigenados, alcoholes: linalol, mentol, geraniol, nerol y sus acetatos; cetonas: carvona, mentona, verbenona; aldehídos: citral (geranial y neral, sus óxidos); hidrocarburos monoterpénicos: pinenos, ocimenos, carenos, terpinenos, limoneno, $p$-cimeno; se encuentran también sesquiterpenoides, entre los cuales se pueden mencionar cariofileno, farneseno, bisaboleno, cadineno, cubebeno, elemeno, germacreno y sus isómeros estructurales; óxidos y alcoholes, e.g., óxido de cariofileno, alcoholes farnesol, nerolidol y sus ésteres. La familia más grande en la fragancia floral, tal vez, está conformada por los hidrocarburos, alifáticos, $\mathrm{C}_{1}-\mathrm{C}_{30}$ (más frecuentes, $\mathrm{C}_{13}-\mathrm{C}_{21}$ ) y olefínicos y algunas cicloparafinas. Estas sustancias, junto con los ácidos grasos, $\mathrm{C}_{12}-\mathrm{C}_{22}$, forman parte de la capa protectora de cera que reviste los pétalos de muchas flores. Una nota odorífera distintiva en la fragancia floral se debe a la presencia en sus mezclas de compuestos que contienen átomos de azufre o nitrógeno, provenientes probablemente del metabolismo de aminoácidos; entre estos metabolitos secundarios volátiles, figuran compuestos con grupo nitro, indoles, oximas, nitrilos, antranilatos y sulfuros, entre los más comunes.

\section{Métodos de obtención de fragancias florales}

Antes de proceder a recolectar los volátiles de flores, es importante establecer si su monitoreo se hará in vivo (en el campo) o ex vivo. El montaje experimental para cada propósito será diferente. Algunas técnicas de extracción no son aplicables en el campo para monitoreo de flores in vivo. Es importante también tener conocimiento previo sobre la cantidad de volátiles que emite la flor, ya que de la cantidad dependerá el sistema de extracción y concentración empleado. El buqué de sustancias volátiles producidas por las flores puede tener de uno a más de cien compuestos, pero, generalmente, contiene de 20 a 60 sustancias diferentes ${ }^{[1]}$. La cantidad de volátiles emitidos va desde los niveles bajos de picogramos hasta más de $30 \mu \mathrm{g} / \mathrm{L}^{[8]}$. Algunos métodos de recolección de volátiles florales pueden tener un diseño automatizado que permite el monitoreo durante 24 horas o por periodos más largos. Sin embargo, la mayoría de las técnicas de extracción hacen una captura momentánea, un "snapshot", de los volátiles florales emitidos ${ }^{[9,10]}$.

Los métodos de extracción de los metabolitos secundarios volátiles de las flores se pueden dividir en tres categorías grandes, a saber: (I) Técnicas de headspace (headspace, HS, por sus siglas en inglés, o espacio de cabeza) en modos estático o dinámico; (II) Técnicas destilativas, entre ellas, destilación por arrastre con vapor, destilación con agua-vapor, hidrodestilación, hidrodestilación asistida por la radiación 
de microondas y (III) Técnicas extractivas, que emplean disolventes de diferente naturaleza, e.g., grasas (maceración, enfleurage, obtención de pomadas), disolventes apolares (hidrocarburos, obtención de concretos), disolventes polares (alcoholes, obtención de absolutos) y fluidos supercríticos (principalmente, $\mathrm{CO}_{2}$ ). Las técnicas de espacio de cabeza (HS) se emplean en dos "formatos" diferentes: headspace estático (S-HS) y headspace dinámico, e.g., purga y trampa (P\&T). Hoy en día, la técnica más popular para el análisis de fragancias florales es la micro-extracción en fase sólida (SPME, por sus siglas en inglés), operada en espacio de cabeza, HS-SPME ${ }^{[4]}$. Este método de extracción sobre un adsorbente polimérico -que reviste una fibra de sílice fundida-, combina la alta selectividad de extracción, que se logra con la escogencia del polímero, su naturaleza química y el grosor y la optimización de condiciones de muestreo (volúmenes del material y de espacio de cabeza, temperaturas, tiempos de pre-equilibrio y de exposición de la fibra, modos de agitación de la muestra, aditivos, etc.), con la concentración de analitos sobre la fibra. La extracción y la concentración simultánea de la muestra son procesos que destacan a la técnica SPME y la hacen muy ventajosa frente a otros métodos $^{[11-13]}$.

Los métodos headspace aportan la información sobre la composición química de las fracciones volátiles; las técnicas de destilación, sobre aceites esenciales, destilados o condensados, mientras que los métodos extractivos (solventes, $\mathrm{CO}_{2}$ supercrítico), sobre la composición química de mezclas que pueden incluir sustancias de baja volatilidad y mayor masa molecular (>400 Da), que en general se denominan los extractos. Las composiciones de estas mezclas pueden diferenciarse no solamente cuantitativamente, sino cualitativamente. Tal como se mencionó anteriormente, en condensados y extractos prevale- cerán compuestos más "pesados", ácidos grasos, hidrocarburos parafínicos de cadena larga, sus alcoholes o aldehídos, mientras que en las fracciones volátiles, se encuentran compuestos de bajo peso molecular, que -eventualmente- pueden "escapar" durante la destilación, en la etapa de despresurización (SFE- $\mathrm{CO}_{2}$ ) o concentración de los extractos.

La composición química de la fracción volátil de las flores depende tanto de factores intrínsecos (genéticos) de la especie, como de factores extrínsecos, ambientales ${ }^{[14]}$. El hábitat, el ambiente donde crece la planta, las condiciones (temperatura, humedad, luz, tipo de suelo, micronutrientes, etc.), en las cuales se monitorean los metabolitos secundarios florales, afectarán la composición cualitativa y cuantitativa de la fracción volátil emitida y recolectada. Por ello, es muy importante durante la recolección de volátiles florales mantener las condiciones de monitoreo controladas, constantes. Muchísimos factores externos afectarán la producción de volátiles en la flor; entre ellos, figuran los cambios de la temperatura, de la humedad, el aumento o la disminución de la energía lumínica, entre otros. Algunas condiciones de estrés (hídrico, lumínico, nutricional) pueden alterar notoriamente la generación de volátiles florales o hasta suprimir su producción ${ }^{[15]}$.

Algunos aspectos del estudio de las fragancias florales deben contemplar el estado de desarrollo de la flor ${ }^{[16,17]}$. Las flores del árbol de ylang-ylang (Cananga odorata Hook Fil. et Thomson, forma genuina, familia Annonaceae) son materia prima importante para la obtención de aceites esenciales y absolutos, que son ingredientes valiosos en muchos perfumes, jabones, champús, lociones. En el árbol, la flor permanece varias semanas, mientras se madura, inicia con una flor muy pequeña, verde, que aumenta luego su tamaño, permaneciendo varios días verde, 
y, luego, se torna amarilla, grande, con puntos de color marrón. En el mismo árbol, es común encontrar flores en diferentes grados de maduración, junto con los frutos que llevan semillas, a través de las cuales se propaga esta especie vegetal. La composición de volátiles en la flor de ylang-ylang varía notoriamente con su estado de madurez. En flores pequeñas, verdes, se registran 10 veces menos componentes, que en una flor amarilla, madura. En las flores de ylang-ylang maduras, amarillas y completamente desarrolladas, se encuentran 16 y 4 veces más sustancias oxigenadas livianas ( $p$-metilanisol, alcohol bencílico, 1,8-cineol, benzoato y salicilato de metilo y etilo, linalol, nerol, geraniol, acetato de bencilo, anetol, acetato de cinamilo, otros) y sustancias oxigenadas más pesadas (sesquiterpenoles, farnesal, farnesol, nerolidol y sus acetatos, cedrol, benzoato y salicilato de bencilo, otros), que en las flores verdes, pequeñas, que empiezan su desarrollo. Los compuestos nitrogenados, fenilacetonitrilo, 4-metilbenzaldoxima, indol, 2-fenil-1-nitroetano y antranilato de metilo, solo aparecen en las flores maduras, grandes y amarillas ${ }^{[18,19]}$.

La composición de los metabolitos secundarios en la emisión floral varía también según la parte de la flor, de donde se extraen los volátiles. En los pétalos de las flores de ylang-ylang, prevalecen los compuestos oxigenados (monoterpenos oxigenados, bencenoides y fenilpropanoides), mientras que en los ovarios (parte central de la flor, pequeña y compacta) abundan los hidrocarburos monoterpénicos y sequiterpénicos ${ }^{[20]}$. La composición porcentual relativa de las familias de compuestos presentes en las flores de ylang-ylang depende del método de su extracción: la destilación con vapor o la destilación-extracción con solvente simultánea (SDE, por sus siglas en inglés) permiten obtener mezclas de metabolitos secundarios, ricas en compuestos oxigenados livianos (50-60\%) y en compuestos oxigenados más pesados (18-20\%), mientras que la extracción con fluido supercrítico, SFE- $\mathrm{CO}_{2}$ aísla extractos, ricos en hidrocarburos alifáticos $\left(C_{n}>20\right)$ y terpénicos, compuestos nitrogenados $\mathrm{y}$ hasta algunos ácidos grasos $\left(\mathrm{C}_{14}-\mathrm{C}_{18}\right)^{[19]}$.

El perfil de compuestos volátiles emitidos por la flor depende también de la hora del día; los insectos que la polinizan pueden ser diurnos o nocturnos, y, de ello, dependerá también la cinética de emanación de compuestos fragantes y el tipo de los volátiles emitidos por la flor, que varían, para la mayoría de las flores, con la hora del día (ritmo circadiano) y según la función biológica que cumplen. Por ejemplo, en las flores de ylang-ylang, la cantidad de sustancias nitrogenadas cambia durante el día: es máxima en la madrugada, disminuye después del mediodía y vuelve a aumentar en las horas de la tarde y de la noche (Figura 1).

La fragancia de flores de Brugmansia suaveolens (Familia Solanácea) sigue un claro ritmo circadiano: la emisión de volátiles se incrementa por la tarde y alcanza su máximo a las nueve de la noche; luego, la emisión de volátiles empieza lentamente a disminuir (Figura 2); en la mañana y durante el día, las flores casi no huelen, aunque atraen masivamente las abejas ${ }^{[3]}$. Es interesante anotar, que algunas flores cambian su fragancia después de haber sido polinizadas; esto sucede con las flores de algunas orquídeas (Ophrys sphegodes) $^{[21,22]}$.

En la Tabla 1, se reúne la información sobre las sustancias volátiles emitidas por las flores de diferentes especies vegetales, provenientes de distintos orígenes (África Oriental, Asia, Arabia, América del Sur, Centroamérica o México) y cultivadas en el Complejo Agroindustrial Piloto de CENIVAM (Bucaramanga, Colombia). Los volátiles se obtuvieron por HS-SPME, usando la fibra de PDMS/DVB $(65 \mu \mathrm{m})$, expuesta a las flores frescas (ca. $20 \mathrm{~g}$ ), recién cortadas, colo- 


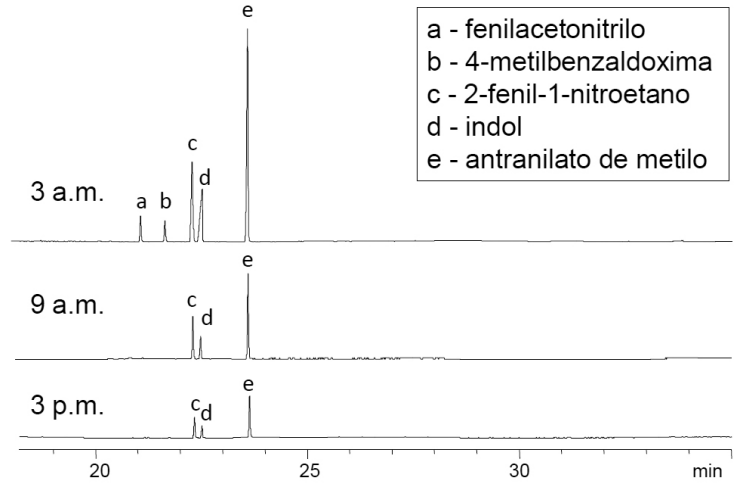

Figura 1 Cromatograma típico de los metabolitos secundarios volátiles de flores de ylang-ylang (solo se registran compuestos nitrogenados), monitoreados a diferentes horas del día. Columna DB-1 (60 m). Detector selectivo de nitrógeno y fósforo, NPD.

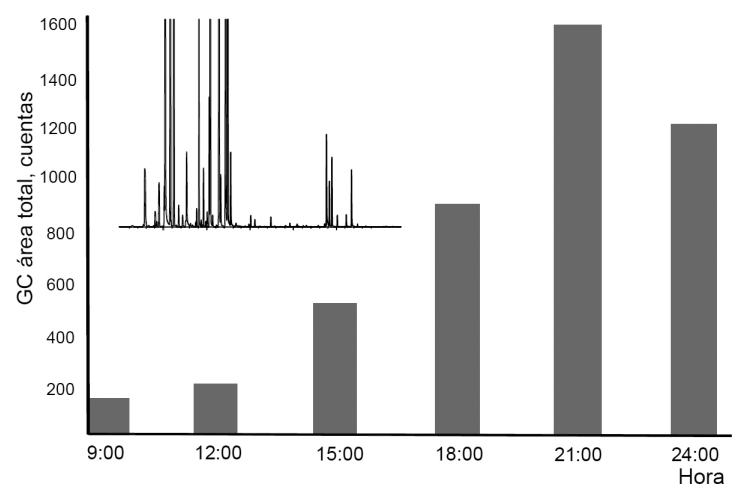

Figura 2 Perfil cromatográfico de los metabolitos secundarios volátiles de flores de Brugmansia suaveolens (familia Solanácea), aislados por HS-SPME, y el cambio de la cantidad de volátiles emitidos por la flor (área cromatográfica total, GC-FID), en función de la hora del día.

cadas en un recipiente sellado $(22 \mathrm{~mL})$, a $40{ }^{\circ} \mathrm{C}$ durante $30 \mathrm{~min}$, con el tiempo de pre-equilibrio de $15 \mathrm{~min}$. En experimentos previos, se ha determinado que las mejores recuperaciones (área cromatográfica total más alta) de volátiles se obtienen, en su orden creciente, con las fibras, así: PA $(85 \mu \mathrm{m})<$ PDMS $(100 \mu \mathrm{m})<$ PDMS/DVB $(65 \mu \mathrm{m}) \approx \mathrm{CAR} / \mathrm{PDMS}(65 \mu \mathrm{m})$. Con tiempos de exposición de la fibra cortos (2-5 min), solo se logra recuperar aldehídos y alcoholes volátiles e hidrocarburos monoterpénicos; con tiempos de exposición más prolongados (20-40 min), en la fracción volátil, se registran también bencenoides, fenilpropanoides, monoterpenos oxigenados y sesquiterpenoides, junto con parafinas $\left(C_{n}>13\right)$ y sus análogos insaturados, aldehídos $y$ alcoholes de cadena más larga $\left(\mathrm{C}_{\mathbf{n}}>13\right)$; sustancias nitrogenados, indoles y antranilatos. A pesar de que las flores pertenecen a plantas de géneros diferentes, varios componentes volátiles en sus emisiones florales son los mismos (Tabla 1). La diversidad en la composición se aprecia en que entre los 115 compuestos diferentes que aparecen en la Tabla 1, el 51\% de los compuestos aparece en solamente 1 de las especies, el $31 \%$ aparece en 2 especies, $10 \%$ en 3, 3,5\% en 4 y 3,5\% en 5 especies. En una comparación hecha entre 165 artículos científicos sobre composición de fragancias florales, se encontró que la rosa fue la flor más estudiada (21 publicaciones), seguida por la petunia (10) y el jazmín (6), entre 73 flores diferentes objeto de estos estudios ${ }^{[2]}$. El linalol fue el constituyente volátil encontrado más frecuentemente (24 especies), seguido del 2-feniletanol (19 especies), el geraniol, el $\beta$-ocimeno, el germacreno D y el citronelol (9 especies). La microextracción en fase sólida fue la técnica de aislamiento de sustancias volátiles más común en estos artículos (33\%), seguida del muestreo headspace (22\%), la extracción con solventes (16\%) y la destilación (13\%).

\section{Análisis cromatográfico de fragancias florales}

Las sustancias que conforman la fracción volátil aislada de las flores son de bajo peso molecular (<300 Da) y conforman mezclas de componentes con diversa polaridad y concentración; gracias a la naturaleza de los compuestos volátiles, su análisis se hace por cromatografía de gases (GC). Debido a la complejidad de algunas 


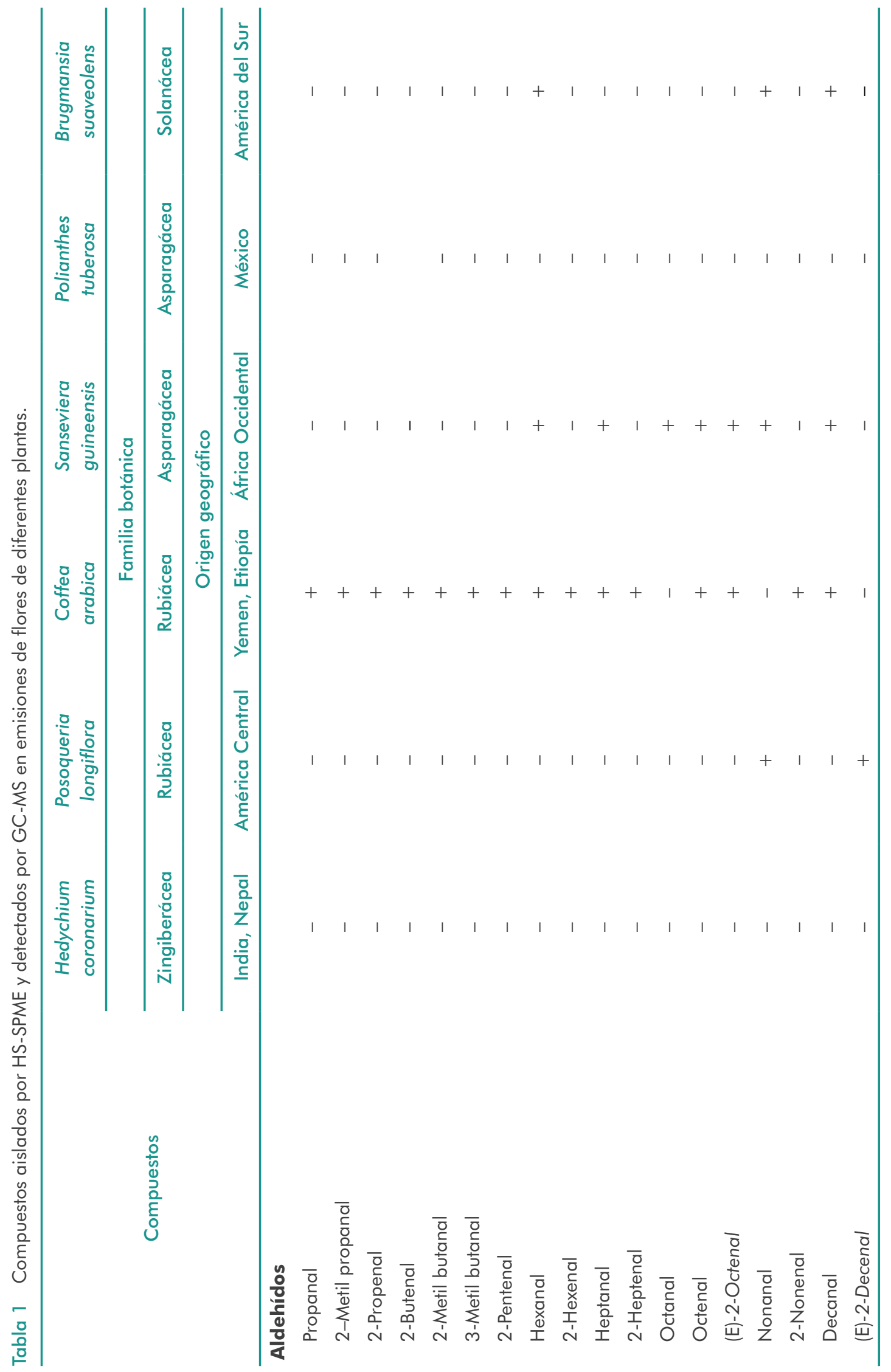




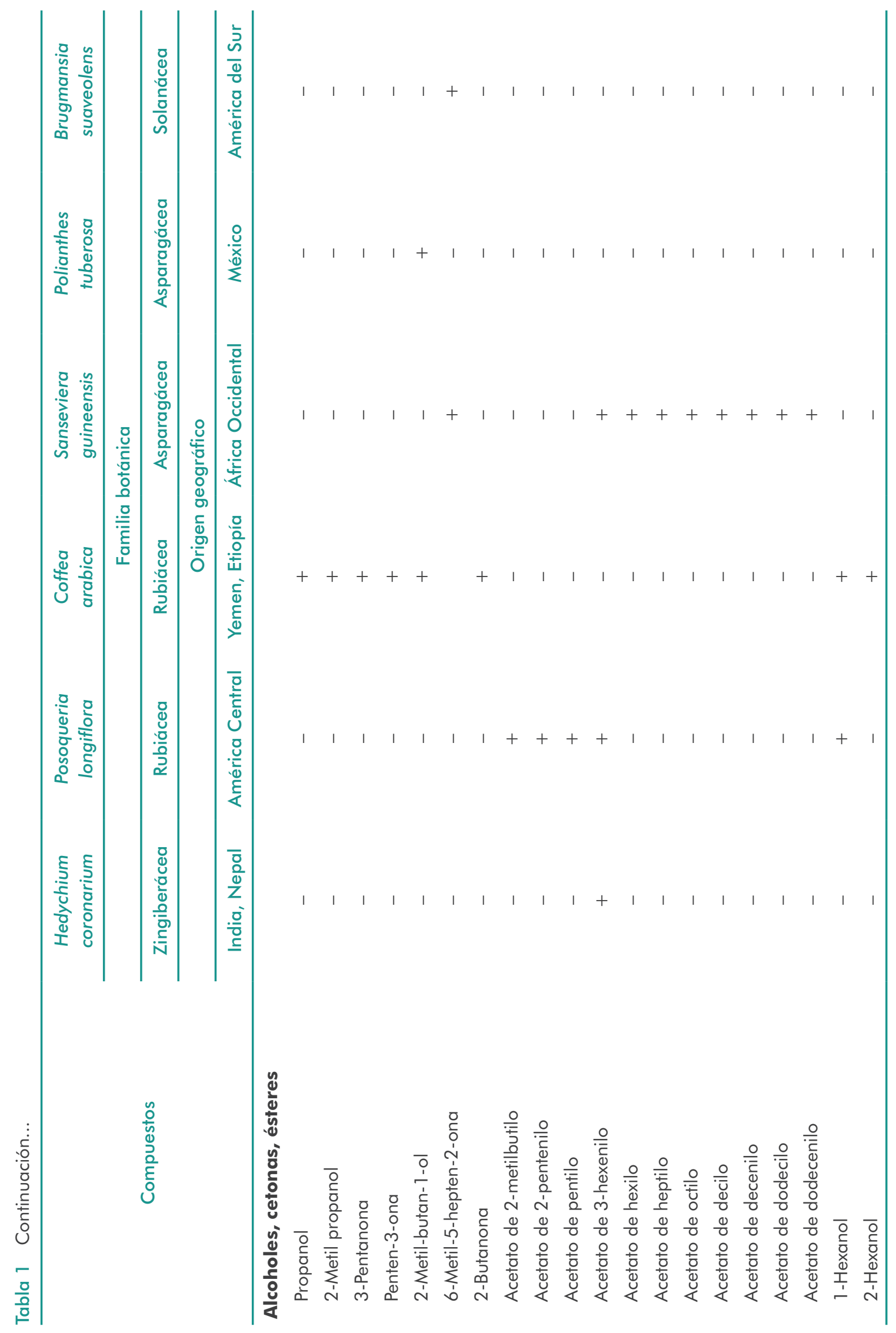




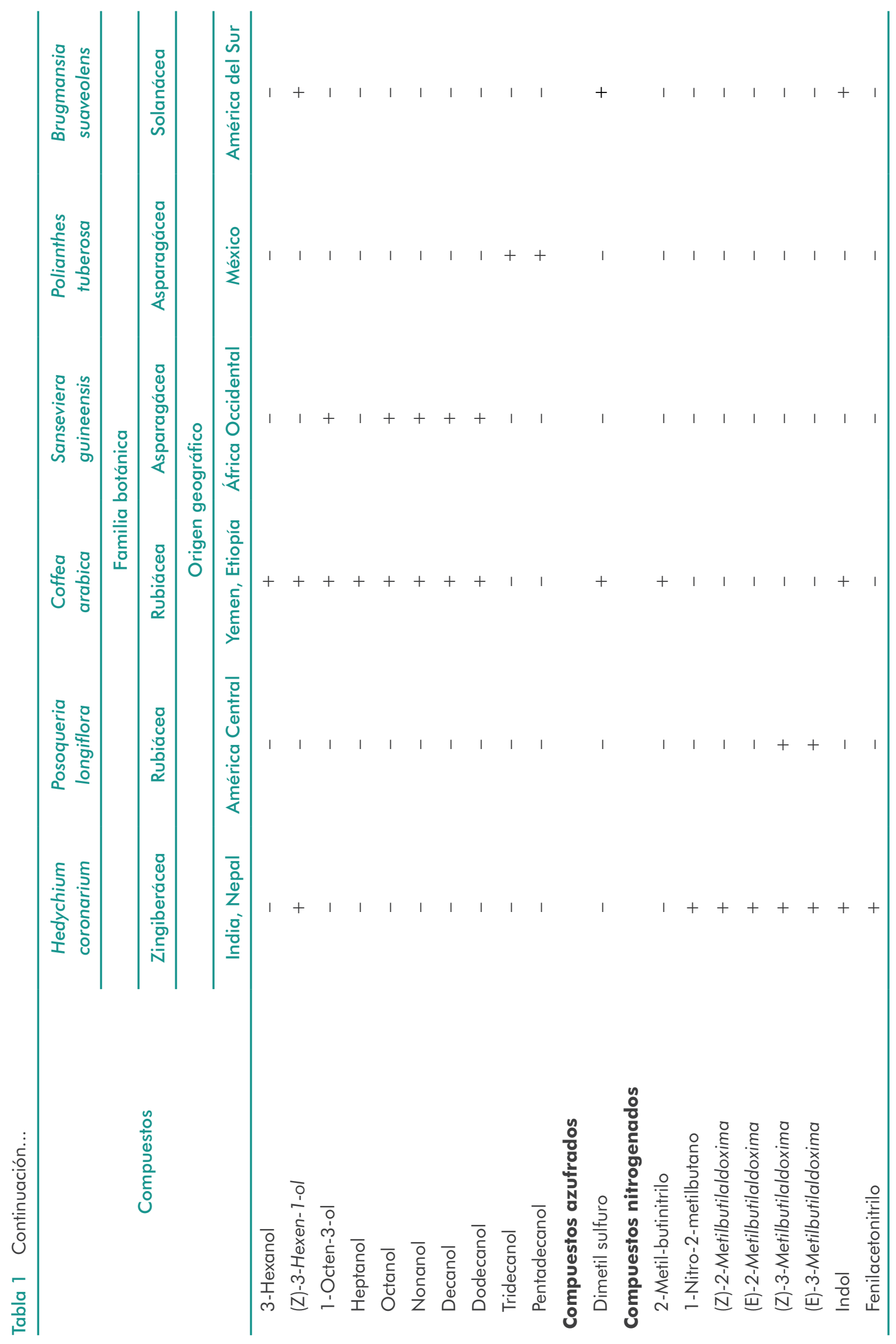




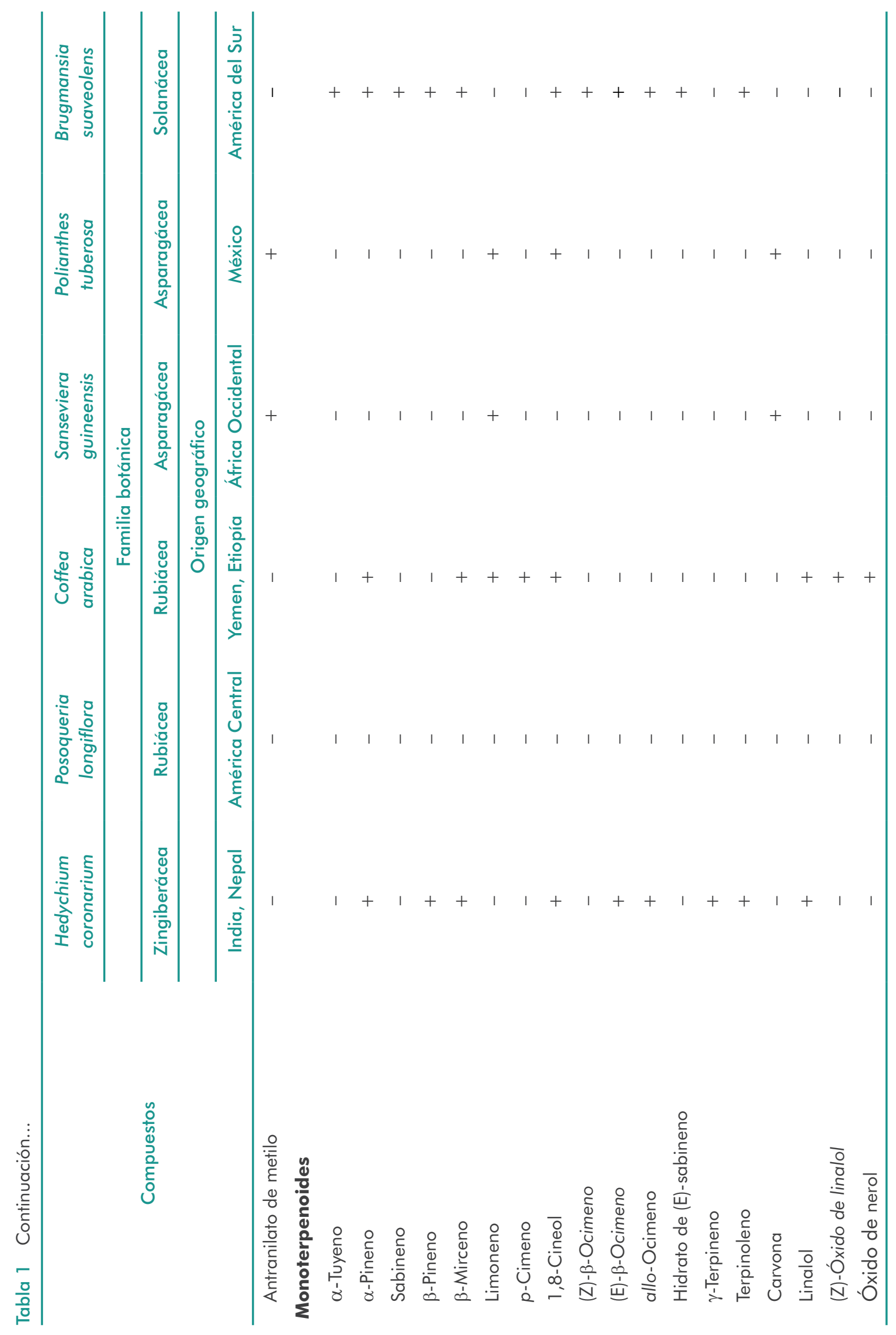




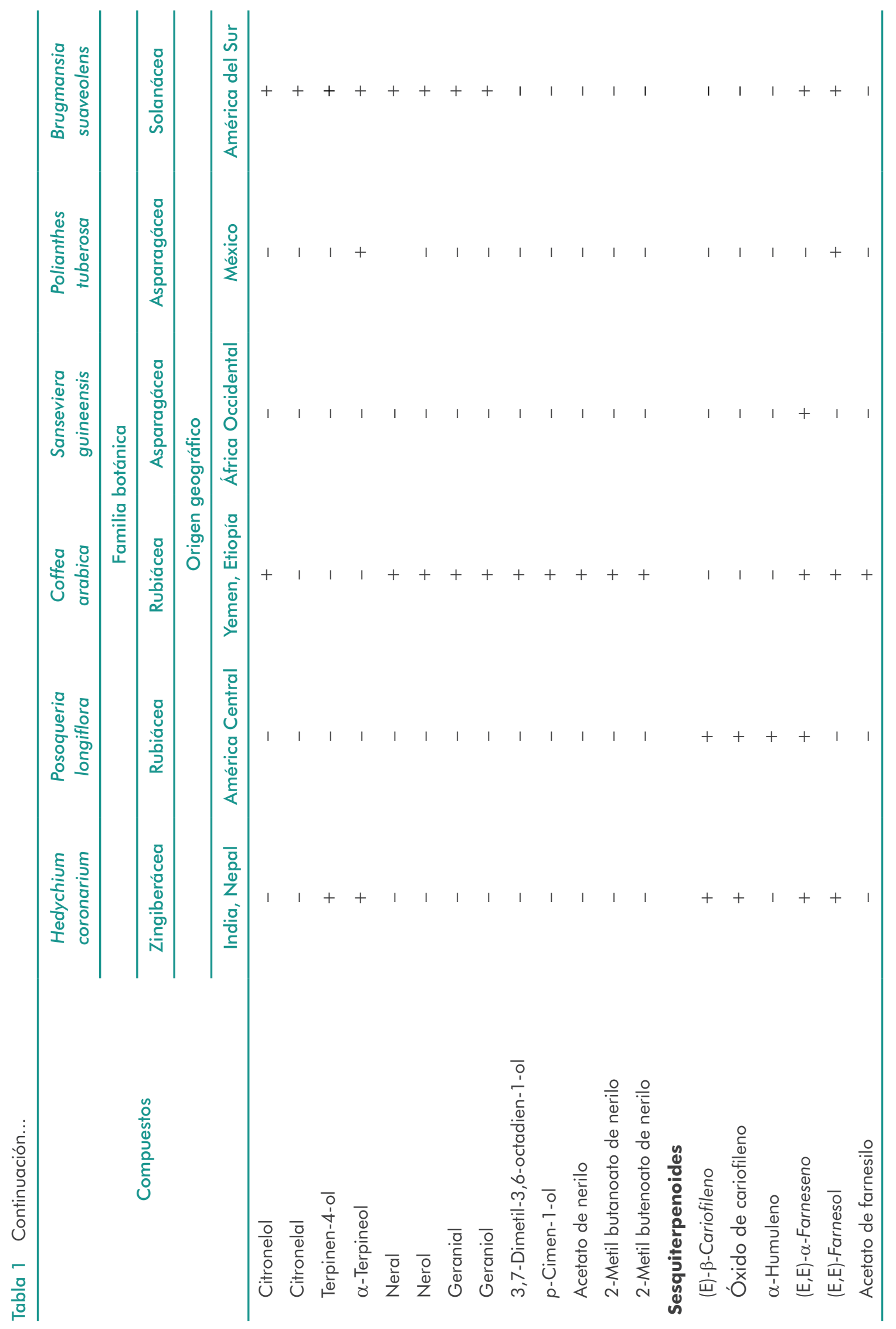




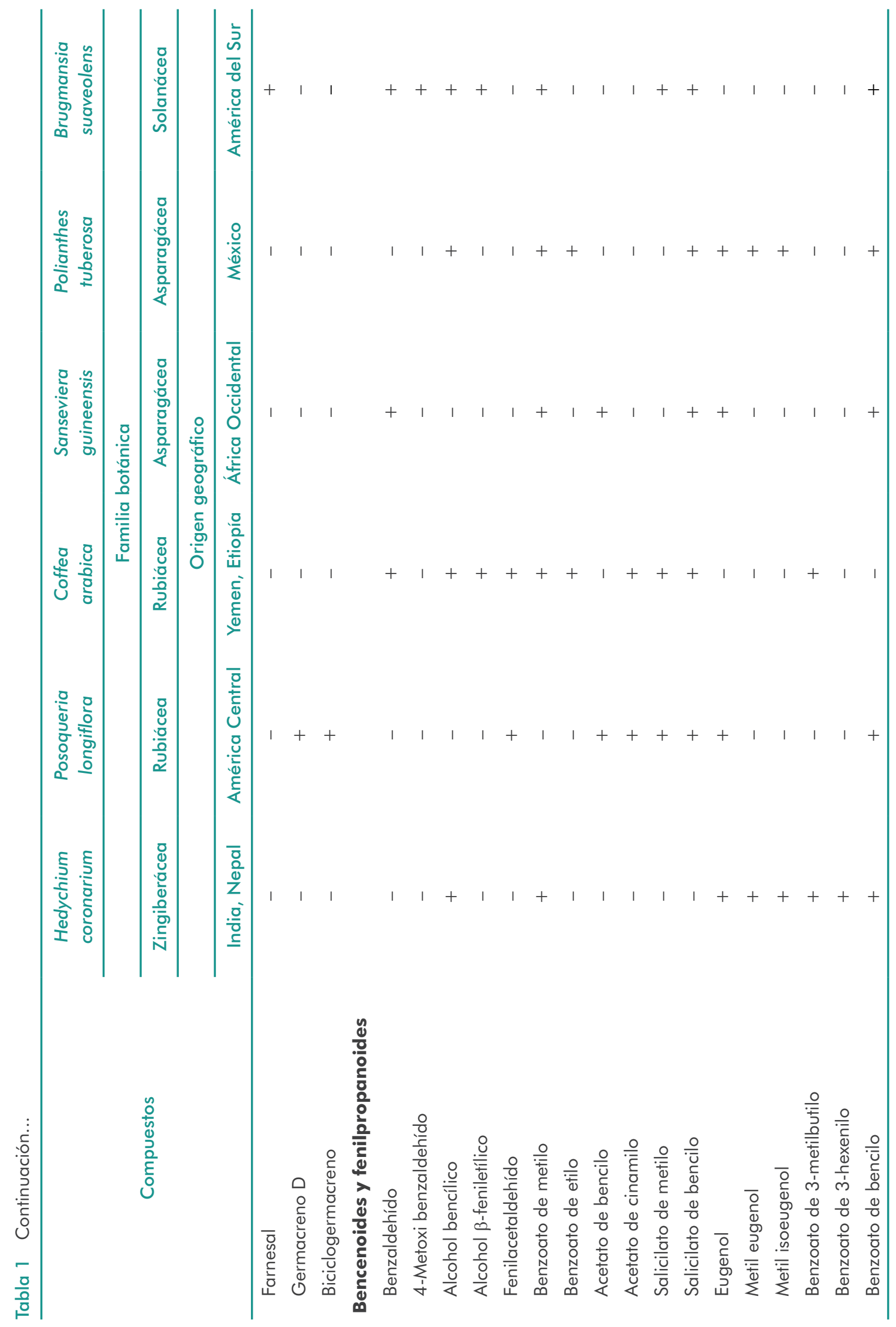


mezclas de volátiles aislados de flores y a la presencia en ellas de sustancias isoméricas (isomería geométrica, de posición, estereoisomería), se recomienda hacer su análisis en columnas capilares de sílice fundida, preferiblemente largas, de 50 o $60 \mathrm{~m}$, con diámetros internos (D.I.) de 0.25 , 0.22 o $0.20 \mathrm{~mm}$. Los diámetros internos menores, aunque permiten aumentar la resolución, eventualmente, pueden comprometer también la sensibilidad. Se usan columnas con el grosor de la fase estacionaria $\left(d_{\mathrm{f}}\right)$ igual o mayor de $0.25 \mu \mathrm{m}$, todo ello, para que la forma de picos, su separación y la sensibilidad, necesaria para su detección reproducible, sean adecuadas.

Generalmente, para la inyección de la muestra $\left(\mathrm{T}^{\circ}\right.$ del inyector, usualmente, de $\left.230-250^{\circ} \mathrm{C}\right)$, se puede usar la relación de split de 1:30, pero, cuando las concentraciones de algunos componentes de interés son bajas, es conveniente la inyección en modo splitless. Cuando se emplea el modo de inyección splitless, para disminuir la "dispersión" o el ensanchamiento de los picos de sustancias muy volátiles, se puede hacer la inyección en el modo de splitless pulsado; es cuando la presión de entrada del gas de arrastre, durante la transferencia de la muestra por el liner, se incrementa unas 2-3 veces. Teniendo en cuenta la presencia de algunas sustancias termolábiles, la inyección on-column o con la programación de temperatura (con PTV, programmed-temperature vaporizer, por sus siglas en inglés), podría ser una buena alternativa.

Para el análisis de las fracciones volátiles, la temperatura inicial de la columna de $35-50^{\circ} \mathrm{C}$ sería la recomendable; la naturaleza de la muestra (compuestos volátiles) no exige que la temperatura final de la columna sea alta, la de $200-250^{\circ} \mathrm{C}$ será suficiente para eluir los componentes más retenidos. La velocidad de calentamiento de la columna es función de su longitud: a mayor longitud, más lentamente hay que calentar la colu- mna, $3-4^{\circ} \mathrm{C} / \mathrm{min}$, pero si se usan columnas más cortas, e.g., de $30 \mathrm{~m}$, se podría ir incrementando la temperatura de la columna más rápidamente, a razón de $5-7^{\circ} \mathrm{C} / \mathrm{min}$. Por supuesto, el proceso de programación de la temperatura en la columna es optimizable, depende de la complejidad de la mezcla de sustancias a analizar (número de componentes, isomería o similitud estructural), su naturaleza (polaridad, peso molecular), las dimensiones de la columna (L, D.I.), el tipo de la fase estacionaria (polaridad) y su grosor $\left(\mathrm{d}_{\mathrm{f}}\right)$.

Para el análisis de la fracción volátil floral se usan, en combinación, dos columnas: una, con la fase estacionaria polar, poli(etilenglicol) (e.g., INNOWAX, DB-WAX, HP-20M, otras) y la otra, con la fase estacionaria apolar, poli(dimetilsiloxano) (HP-1, Ultra 1, DB-1, BP-1, otras) o 5\%-fenil poli(dimetilsiloxano) (HP-5, DB-5, Ultra 2, CPSil 5, BP-5, otras). En la Figura 3, se pueden observar perfiles cromatográficos de la fragancia de flores de café (Coffea arabica, Fam. Rubiácea), aislada por HS-SPME (CAR/PDMS, $65 \mu \mathrm{m}$; temperatura $-60^{\circ} \mathrm{C}$, tiempo de exposición de la fibra - $30 \mathrm{~min}$ ), obtenidos por GC-MS en columnas de diferente polaridad.

La cromatografía de gases enantioselectiva aprovecha el hecho de que los enantiómeros poseen tiempos de retención diferentes cuando en la fase estacionaria se insertan compuestos que pueden formar aductos cuya estabilidad es función de la forma tridimensional del analito. Las ciclodextrinas, con su geometría de cono con cavidad de diferente tamaño, han resultado ser agentes quirales muy efectivos al constituir complejos de inclusión que permiten discriminar isómeros según su forma. Las fragancias de jazmín (Jasminum grandiflorum) y otras flores (Osmanthus fragrans, Boronia megastima) contienen una mezcla de estereoisómeros del jasmonato de metilo. Wilfred König reportó la separación de todos los isómeros por medio de 

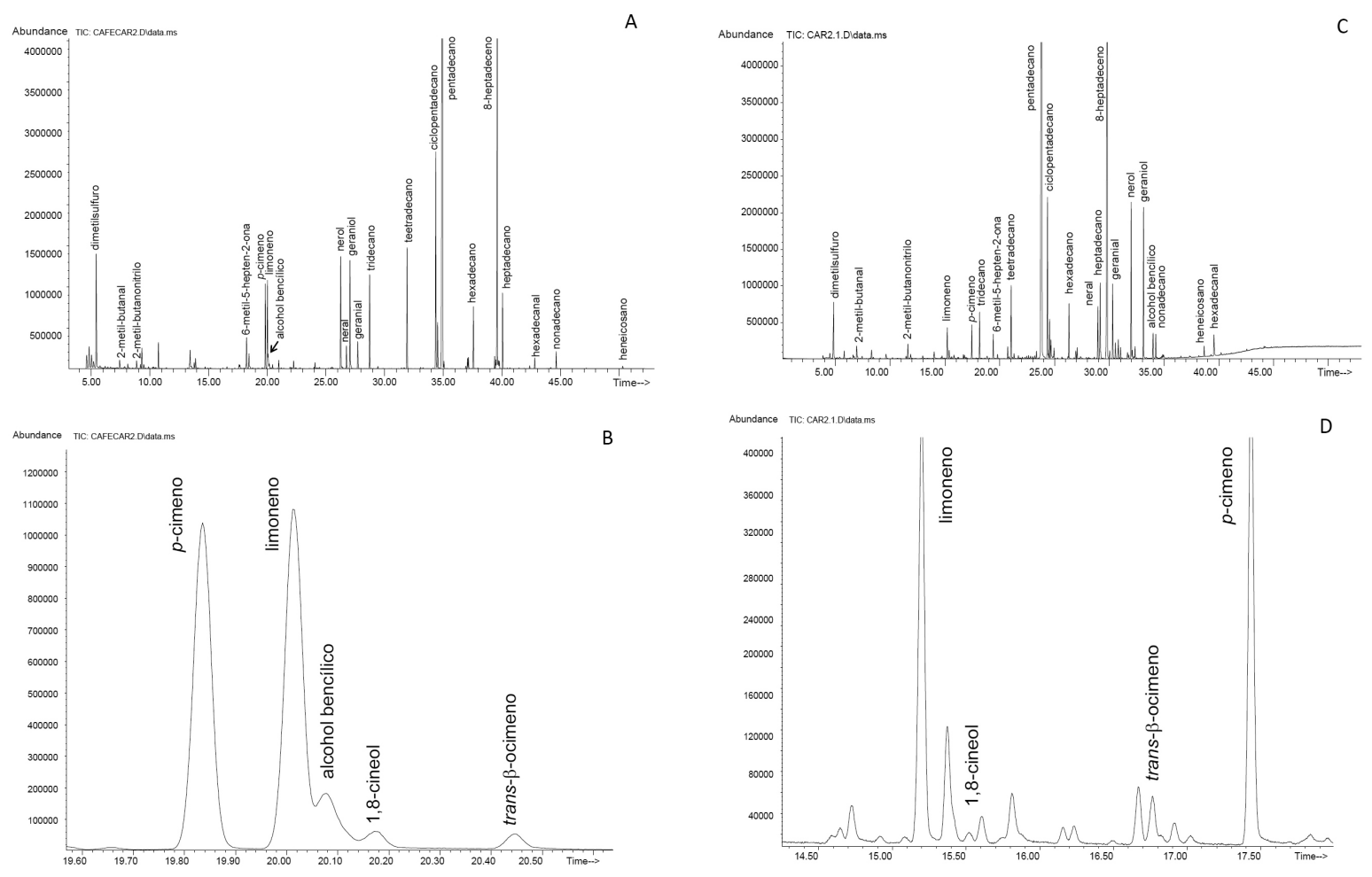

Figura 3 Cromatogramas típicos de la fragancia de flores de café aislada por HS-SPME (CAR/PDMS, $65 \mu \mathrm{m}$; $60^{\circ} \mathrm{C}$, tiempo de exposición de la fibra - $\left.30 \mathrm{~min}\right)$. A. Columna cromatográfica apolar DB-5MS $(60 \mathrm{~m} \times 0.25 \mathrm{~mm}$ x $0.25 \mathrm{~mm}$ ). Detector selectivo de masas (MSD, El, $70 \mathrm{eV}$ ); B. Fragmento ampliado del mismo cromatograma, donde se observa la separación deficiente de limoneno $(L R I=1030)$, alcohol bencílico $(L R I=1031)$ y 1,8-cineol $(\mathrm{LRI}=1032)$; C. Columna cromatográfica polar DB-WAX (60 m x $0.25 \mathrm{~mm} \times 0.25 \mu \mathrm{m})$. Detector selectivo de masas (MSD, El, $70 \mathrm{eV}$ ); D. Fragmento ampliado del mismo cromatograma, donde se observa la separación mejor de limoneno y 1,8-cineol; el alcohol bencílico tiene un tiempo de retención mucho más largo $\left(\dagger_{\mathbf{R}}=34.1\right.$ $\min , \mathrm{LRI}=1895$ ) en la columna polar y no co-eluye con limoneno, tal como sucede en la columna apolar (B).

cromatografía de gases preparativa en la que utilizó columnas empacadas con ciclodextrinas ${ }^{[23]}$. Esto permitió confirmar la estimación hecha por Acree y Barnard, de que el isómero (+)-epi-jasmonato de metilo tiene un umbral de olor unas 500 veces más bajo que el del isómero mayoritario, el (-)-jasmonato de metilo ${ }^{[24]}$.

Los aldehídos y alcoholes lilac son monoterpenos oxigenados que se encuentran en especies vegetales de muchas familias. Lamiaceae (Origanum vulgare ${ }^{[25]}$ ), Orchidaceae (Platanthera sp. ${ }^{[26]}$ ), Rosaceae (Prunus padus ${ }^{[27]}$ ), y Rubiaceae (Cephalanthus occidentalis ${ }^{[25]}$ ) son algunos ejemplos de estas familias. Cada una de las moléculas lilac posee 3 carbonos quirales, lo que da lugar a 8 estereoisómeros del aldehído y 8 estereoisómeros del alcohol lilac. Dötterl et al. ${ }^{[28]}$ lograron separar todos los isómeros del aldehído y 7 isómeros del alcohol lilac, por medio de un sistema de cromatografía de gases bidimensional en el que una columna capilar de $30 \mathrm{~m}$ con fase estacionaria de 5\%-fenil polimetilsiloxano fue unida por medio de una válvula $\mathrm{T}$ a un detector selectivo de masas y a otra columna capilar de $30 \mathrm{~m}$ con una fase estacionaria formada por fenil-poli(dimetilsiloxano) (70\%) y un derivado de ciclodextrina (30\%). Cada columna tuvo un horno independiente. Este sistema se modificó para convertirlo en cromatografía micropreparativa. La salida de la segunda columna se conectó 
a un divisor de flujo que permitió dirigir una parte del efluente hacia un detector de ionización en llama y la otra parte hacia una barra de agitación recubierta de PDMS, para absorber los analitos separados. Esta modificación permitió recoger los isómeros, que luego se emplearon en experimentos de electroantenografía en los que se utilizaron antenas de diferentes insectos para examinar si había alguna respuesta selectiva para alguno de los isómeros. Se encontró que las antenas de la polilla Hadena bicruris respondieron a los 8 isómeros de aldehído lilac, pero fueron más sensibles a algunos isómeros que a otros.

El sistema de detección más común para el análisis comparativo y para la cuantificación de compuestos en las fracciones volátiles aisladas de flores es el detector de ionización en llama (FID), un sistema simple, robusto, con una sensibilidad aceptable y un amplio rango dinámico. Los detectores selectivos, tales como el detector de nitrógeno y fósforo (NPD) y el detector fotométrico de llama (FPD), son herramientas muy útiles para la detección selectiva de compuestos nitrogenados y azufrados, muy frecuentes en las fragancias florales.

Un ejemplo interesante de la aplicación de la antenografía al estudio de la relación planta-insecto es el trabajo de Balao et al. ${ }^{[29]}$, sobre la emisión de volátiles por las flores de Dianthus inoxianus. Esta especie es polinizada únicamente durante la noche por solamente un tipo de polinizador, Hyles livornica, un insecto que poliniza un buen número de especies diferentes. La esencia floral de $D$. inoxianus contiene 68 compuestos volatiles, pero está dominada por sustancias alifáticas que poseen un grupo cetona en la posición 2 . El análisis cromatográfico mostró que la cantidad total de volátiles liberados durante el día es similar a la de la noche, lo cual no se correlaciona con la actividad del insecto polinizador. Sin embargo, la electroantenografía permitió determinar cuáles compuestos volátiles causan respuesta en las antenas de machos y hembras de H. livornica. Al seguir su variación entre día y noche, se encontró que la proporción de compuestos activos aumenta en la noche, lo que sirve de señal para atraer al insecto polinizador.

El ejemplo anterior muestra un tipo de experimento que solamente es posible al disponer de un detector de alta especificidad. Empero, el sistema de detección más importante y de mayor uso en el análisis de mezclas volátiles es el detector selectivo de masas (MSD); su combinación con la cromatografía capilar (GC-MS) es un instrumento perfecto para lograr la separación e identificación (presuntiva o confirmatoria) de componentes presentes en una mezcla.

El modo de ionización más empleado para el análisis de sustancias volátiles es el impacto con electrones (EI) de $70 \mathrm{eV}$ de energía. Los espectros de masas (MS) de EI contienen mucha información, porque en el espectro aparecen señales de numerosos fragmentos ionizados, que forman una combinación única y que permite diferenciar una molécula de la otra. En la Figura 4, aparecen espectros de masas de dos sesquiterpenos, $\beta$-cariofileno y $\alpha$-humuleno, presentes frecuentemente en flores y plantas en general. La masa $(\mathrm{m} / z)$ de fragmentos (iones o ion-radicales) y sus abundancias relativas, que conforman el patrón de fragmentación, son la guía para diferenciar las estructuras. Los índices de retención lineales (LRI, por sus siglas en inglés), de ambos sesquiterpenos se diferencian en 15-20 unidades para columnas de fases estacionarias, polar y apolar. Los LRI medidos experimentalmente en las columnas polar y apolar, se comparan con los registrados en la literatura o en bases de datos ${ }^{[30-35]}$.

Sin embargo, hay situaciones más complejas: es cuando los espectros de masas y LRI de las sustancias son similares y el margen de duda 

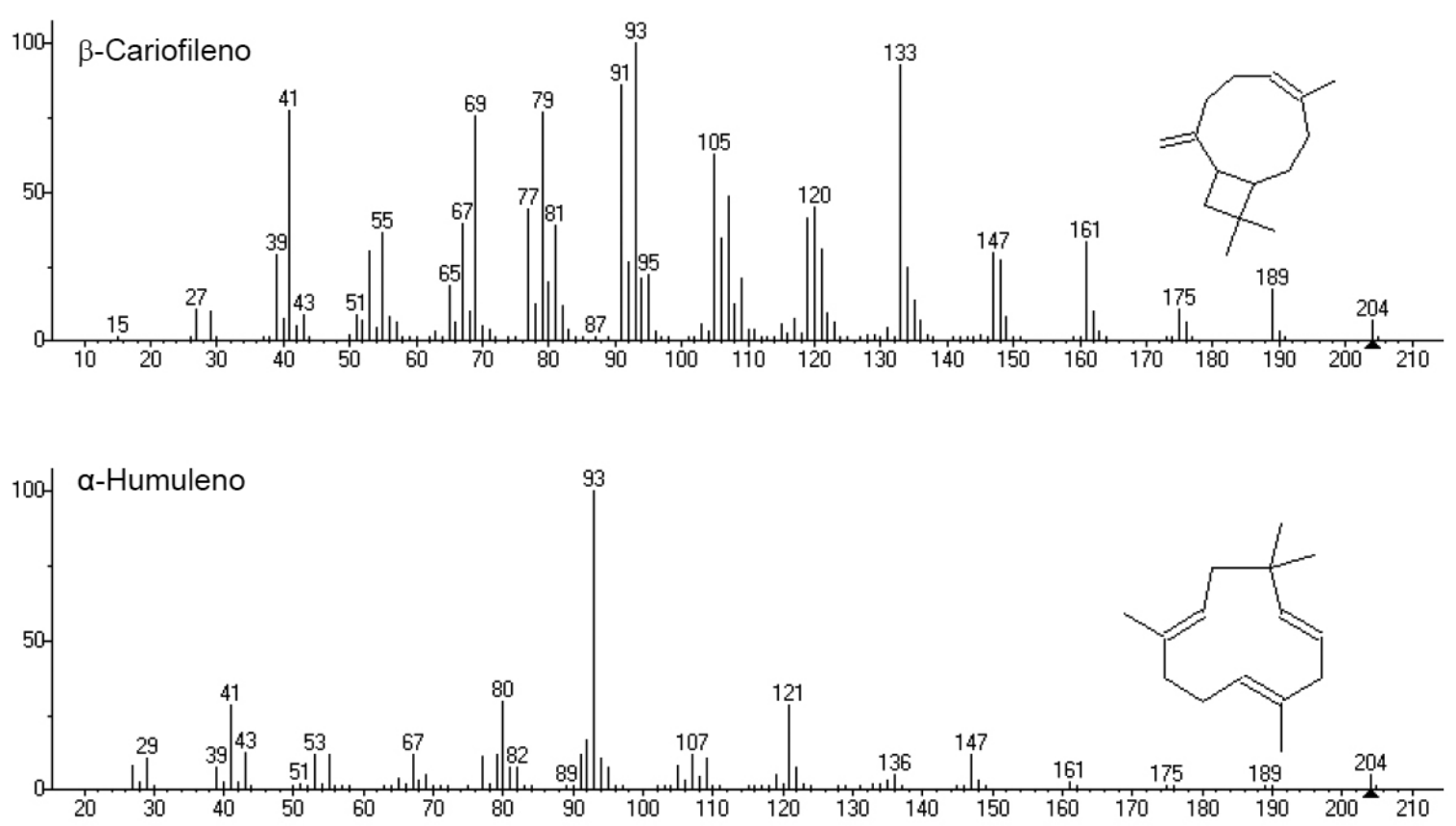

Figura 4 Espectros de masas de impacto con electrones ( $\mathrm{El}, 70 \mathrm{eV}$ ) de los sesquiterpenos isoméricos, $\beta$-cariofileno y $\alpha$-humuleno, que presentan patrones de fragmentación claramente distinguibles [Tomado de: NIST Chemistry WebBook, NIST Standard Reference Database Number 69, hHtp://webbook.nist.gov]. Sus índices de retención lineales (LRI) se diferencian en 15-20 unidades en columnas apolares, e.g., DB-1 [poli(dimetilsiloxano)] o DB-5 [5\%-fenil poli(metilsiloxano) $]^{[31,34]}$, lo que ayuda a diferenciarlos por su retención en la columna, apolar o polar.
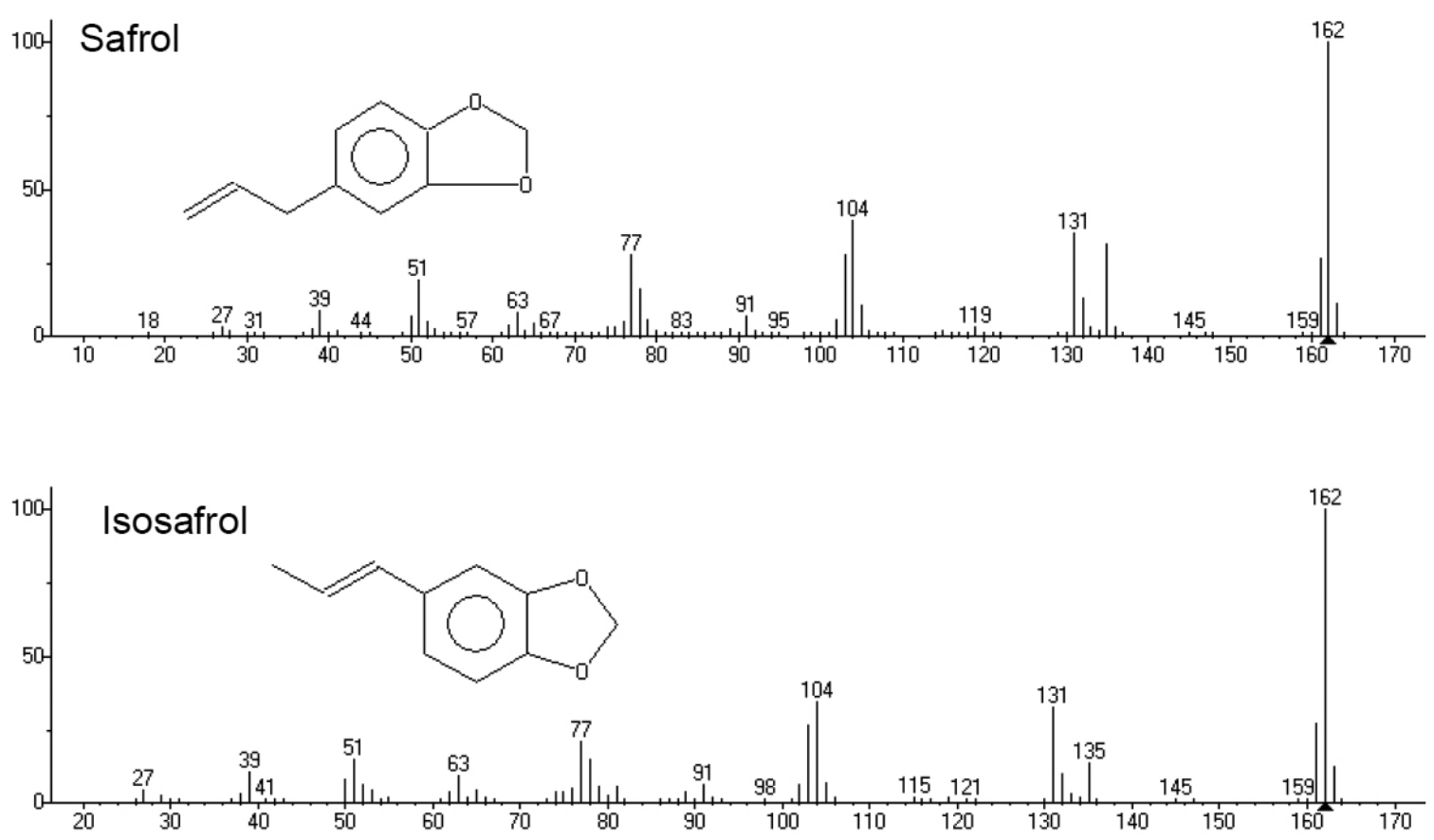

Figura 5 Espectros de masas de impacto de electrones (El, $70 \mathrm{eV})$ de safrol e isosafrol, que presentan patrones de fragmentación muy similares [tomado de: NIST Chemistry WebBook, NIST Standard Reference Database Number 69, http://webbook.nist.gov], que no permiten diferenciarlos solo con base en sus espectros de masas. 
es bastante amplio. En la Figura 5, aparecen los espectros de masas (EI, $70 \mathrm{eV}$ ) de safrol y de su isómero, isosafrol. El isosafrol puede existir en dos formas geométricas, isómero cis- e isómero trans-. Es difícil diferenciar las dos sustancias por sus espectros de masas: solo se observan pequeñas diferencias cuantitativas en las abundancias de algunos iones. Los LRI de los isómeros no se diferencian mucho, en unas 5-7 unidades, lo que no permite diferenciarlos con base en este parámetro ${ }^{[36]}$ : en este caso, para la identificación confirmatoria del compuesto se requiere el uso de una sustancia certificada (patrón).

En el caso de estudio de emisiones florales y la relación de algunos componentes como atrayentes de polinizadores, la identificación confirmatoria es importante, pero para muchas sustancias, no hay patrones comerciales; entonces, se recurre a la síntesis de la sustancia, para su identificación química completa.

El análisis del aceite esencial de las flores de ylang-ylang por cromatografía completa con analizador de tiempo de vuelo, GCxGC-TOFMS, permitió detectar un total de 161 compuestos, de los cuales 75 no habían sido encontrados en el análisis GC-MS unidimensional ${ }^{[37]}$. Sin embargo, a pesar de que la segunda dimensión cromatográfica permite un aumento dramático en la resolución, algunos compuestos pudieron ser clasificados según su naturaleza química, pero no se logró su identificación completa debido a su gran similitud espectral con otros componentes y a la carencia de sustancias patrón.

En resumen, las fragancias florales son mezclas complejas cuyo estudio requiere una selección cuidadosa de las técnicas de aislamiento y de análisis instrumental. Gracias a los avances en estos campos, es posible ahora lograr una caracterización detallada de los compuestos volátiles emitidos por las flores, hasta distinguir entre diversos estereoisómeros.

\section{Agradecimientos}

Los autores agradecen la financiación del Patrimonio Autónomo Fondo Nacional de Financiamiento para la Ciencia, la Tecnología y la Innovación, Francisco José de Caldas, contrato RC 0572-2012. También agradecen a Martha Cervantes, M.Sc., por su colaboración con búsquedas bibliográficas.

\section{Referencias}

1 Dudareva NA, Pichersky E, editors. Biology of floral scent. Boca Raton: CRC, Taylor \& Francis Group; 2006. 346 p.

2 Scopus. Base de datos. Elsevier; 2013. Consultada en 10-05-2013.

3 Stashenko EE, Martínez JR. Sampling flower scent for chromatographic analysis. Journal of Separation Science 2008; 31:2022-2031. http://dx.doi.org/10.1002/ jssc. 200800151

4 Stashenko EE, Martínez JR. Sampling volatile compounds from natural products with headspace/ solid-phase micro-extraction. Journal of Biochemical and Biophysical Methods 2007; 70:235-242. http:// dx.doi.org/10.1016/j.jbbm.2006.08.011

5 Choi H-S. Characterization of Citrus unshiu (C. unshiu Marcov. Forma Miyagawa-wase) blossom aroma by solid-phase microextraction in conjunction with an electronic nose. Journal of Agricultural and Food Chemistry 2003; 51:418-423. http://dx.doi. org/10.1021/jf0114280

6 KnudsenJT,Eriksson R, GershenzonJ,StåhlB.Diversity and distribution of floral scent. Botanical Review 2006; 72:1-120. http://dx.doi.org/10.1663/00068101(2006)72[1:DADOFS]2.0.CO;2

7 Knudsen JT, Tollsten L, Bergstrom G. Floral scents - a checklist of volatile compounds isolated by headspace techniques. Phytochemistry 1993; 33:253-280. http://dx.doi.org/10.1016/0031-9422(93)85502-I

8 Knudsen JT, Gershenzon J. The chemical diversity of floral scent. In: Dudareva NA, Pichersky E, editors. Biology of floral scent. Boca Raton: CRC, Taylor \& Francis Group; 2006. p. 40.

9 Tholl D, Boland W, Hansel A, Loreto F, Röse U, Schnitzler J-P. Practical approaches to plant volatile analysis. Plant Journal 2006; 45:540-560. http://dx.doi. org/10.1111/j.1365-313X.2005.02612.x 
10 Augusto F, Leite e Lopes A, Zini C. Sampling and sample preparation for analysis of aromas and fragrances. Trends in Analytical Chemistry 2003; 22:160-169. http://dx.doi.org/10.1016/S0165-9936(03)00304-2

11 Barták P, Bednar P, Cáp L, Ondráková L, Stránský Z. SPME - A valuable tool for investigation of flower scent. Journal of Separation Science 2003; 26:715-721. http://dx.doi.org/10.1002/jssc.200301381

12 Flamini G, Cioni PL, Morelli I. Use of solid-phase micro-extraction as a sampling technique in the determination of volatiles emitted by flowers, isolated flower parts and pollen. Journal of Chromatography A 2003; 998:229-233. http://dx.doi.org/10.1016/ S0021-9673(03)00641-1

13 Fernando LN, Grün IU. Headspace-SPME analysis of volatiles of the ridge gourd (Luffa acutangula) and bitter gourd (Momordica charantia) flowers. Flavour and Fragrance Journal 2001; 16:289-293. http://dx.doi. org/10.1002/ffj.999

14 Xiang L, Milc JA, Pecchioni N, Chen L. Genetic aspects of floral fragrance in plants. Biochemistry (Moscow) 2007; 72:351-358. http://dx.doi.org/10.1134/ S0006297907040013

15 Bernhardt P, Sage T, Weston P, Azuma H, Lam M, Thiem $\mathrm{L}$ et al. The pollination of Trimenia moorei (Trimeniaceae): Floral volatiles, insect/wind pollen vectors and stigmatic self-incompatibility in a basal angiosperm. Annals of Botany 2003; 92:445-458. http://dx.doi.org/10.1093/aob/mcg157

16 Effmert U, Große J, Röse U, Ehrig F, Kägi R, Piechulla B. Volatile composition, emission pattern, and localization of floral scent emission in Mirabilis jalapa (Nyctaginaceae). American Journal of Botany 2005; 92:2-12. http://dx.doi.org/10.3732/ ajb.92.1.2

17 Granero AM, Egea Gonzalez FJ, Guerra Sanz JM, Martínez Vidal JL. Analysis of biogenic volatile organic compounds in zucchini flowers: identification of scent sources. Journal of Chemical Ecology 2005; 31:23092322. http://dx.doi.org/10.1007/s10886-005-7103-2

18 Stashenko EE, Torres W, Martínez JR. A study of the compositional variation of the essential oil of ylangylang (Cananga odorata Hook Fil. Et Thomson, forma genuina) during flower development. Journal of High Resolution Chromatography 1995; 18:101-104.

19 Stashenko EE, Quiroz N, Martínez JR. HRGC/FID/ NPD and HRGC/MSD study of Colombian ylangylang (Cananga odorata) oils obtained by different extraction techniques. Journal of High Resolution
Chromatography 1996; 19:353-360. http://dx.doi. org/10.1002/jhrc.1240190609

20 Stashenko EE, Martínez JR, Macku C, Shibamoto T. HRGC and GC-MS Analysis of Essential Oil from Colombian Ylang-Ylang (Cananga odorata Hook Fil. et Thomson, forma genuina). Journal of High Resolution Chromatography 1993; 16:441-444. http:// dx.doi.org/10.1002/jhrc.1240160713

21 Schiestl FP, Ayasse M, Paulus HF, Lofstedt C, Hansson BS, Ibarra $\mathrm{F}$ et al. Orchid pollination by sexual swindle. Nature 1999; 399:421-422. http://dx.doi. org/10.1038/20829

22 Schiestl FP, Ayasse M. Post-pollination emission of a repellent compound in a sexually deceptive orchid: a new mechanism for maximizing reproductive success? Oecologia 2001; 126:531-534. http://dx.doi. org/10.1007/s004420000552

23 König WA. Chirality in the natural world-odours and tastes. In: Lough WJ, Wainer IW, editors. Chirality in Natural and Applied Sciences. Oxford: Blackwell Publishers; 2002. p. 261-84.

24 Acree TE, Barnard J. The analysis of odour-active volatiles in gas chromatographic effluents. In: Shreier P, editor. Analysis of Volatiles. Berlin: Gryter; 1984. p. 251-67.

25 Andersson S, Nilsson L A, Groth I, Bergström G. Floral scents in butterfly-pollinated plants: possible convergence in chemical composition. Botanical Journal of the Linnean Society 2002; 140:129-153. http://dx.doi.org/10.1046/j.1095-8339.2002.00068.x

26 Tollsten L, Bergström G. Fragrance chemotypes of Platanthera (Orchidaceae) - the result of adaptation to pollinating moths? Nordic Journal of Botany 1993; 13:607-613. http://dx.doi. org/10.1111/j.1756-1051.1993.tb00105.x

SurburgH, Güntert M,SchwarzeB. Volatileconstituents of European bird cherry flowers (Padus avium Mill.). Journal Essential Oil Research 1990; 2:307-316. http:// dx.doi.org/10.1080/10412905.1990.9697889

28 Dötterl S, Burkhardt D, Weißbecker B, Jürgens A, Schütz S, Mosandl A. Linalool and lilac aldehyde/ alcohol in flower scents. Electrophysiological detection of lilac aldehyde stereoisomers by a moth. Journal of Chromatography A 2006; 1113:231-238. http://dx.doi.org/10.1016/j.chroma.2006.02.011

29 Balao F, Herrera J, Talavera S, Dötterl S. Spatial and temporal patterns of floral scent emission in Dianthus inoxianus and electroantennographic responses of its hawkmoth pollinator. Phytochemistry 2011; 72:601-609. http://dx.doi. org/10.1016/j.phytochem.2011.02.001 
30 Jennings W, Shibamoto T. Qualitative analysis of flavor and fragrance volátiles by glass capillary gas chromatography. New York: Academic Press; 1980.

31 Davies NW. Gas chromatographic retention indices of monoterpenes and sesquiterpenes on methyl silicon and Carbowax 20M phases. Journal of Chromatography A 1990; 503:1-24. http://dx.doi.org/10.1016/S00219673(01)81487-4

32 Adams RP. Identification of essential oil components by gas chromatography/quadrupole mass spectrometry. Carol Stream: Allured Publishing; 2001.

33 Joulain D, König WA. The atlas of spectral data of sesquiterpene hydrocarbons. Hamburg: E.-B. Verlag; 1998.

34 Acree T, Arn H. Flavornet and human odor space. Available from: http://www.flavornet.org.
35 Pherobase. Database of Pheromones and Semiochemicals. Available from: http://www. pherobase.com.

36 Stashenko EE, Martínez JR. Algunos aspectos prácticos para la identificación de analitos por cromatografía de gases acoplada a espectrometría de masas. Scientia Chromatographica 2010; 2:29-47.

37 Brokl M, Fauconnier M-L, Benini C, Lognay G, Du Jardin P, Focant J-F. Improvement of ylang-ylang essential oil characterization by GC×GC-TOFMS. Molecules 2013; 18:1783-1797. http://dx.doi. org/10.3390/molecules18021783

Recebido: 13/04/2013

Aceito: $21 / 05 / 2013$ 